\title{
Optimization of Current Carrying Muticables using Topological and Shape Sensitivity
}

\author{
Zakaria Belhachmi ${ }^{1}$, Belhassen Meftahi ${ }^{2}$ and Houcine Meftahi*,3 \\ 1 Mathematics, Information Technology and Applications Laboratory (LMIA, EA \\ 3993) 4 rue des Frères Lumié 68093 Mulhouse Cedex France. \\ ${ }^{2}$ Lamsin, Enit of Tunisia, 1002 Tunis. \\ 3 University of Jendouba /Isikef \& Lamsin, Enit of Tunisia, 1002 Tunis.
}

Received November 29, 2018; Accepted June 10, 2019

\begin{abstract}
In this paper, we use the topological and shape gradient framework, to optimize a current carrying multicables. The geometry of the multicables is modeled as a coated inclusions with different conductivities and the problem we are interested is the location of the inclusions to get a suitable thermal environnent. We solve numerically the optimization problem using topological and shape gradient strategy. Finally, we present some numerical experiments.
\end{abstract}

AMS subject classifications: 49Q10, 49Q12, 65K10, 68W25.

Key words: Electrical multicables, heat conduction, shape optimization, topology optimization.

\section{Introduction}

In modern electrical machines like hybrid and electrical cars, manufacturers reduce cable diameters to save material, space and weight. But smaller diameters of the electrical cables result a higher temperatures in the connecting structures. This may cause overheating and irreparable damages of the machines. The heat transfer in the current carrying multi-cables depend on the position of the cables. Therefore finding the positions of cables which lead to the minimal temperature is of interest.

In the sequel, we will use the following notations:

The k-th single cable $C_{k}=\left(x_{k}, y_{k}, r_{k}^{i}, r_{k}^{e}\right)$ is described by its center $\left(x_{k}, y_{k}\right)$, the radius of the current carrying part $r_{k}^{i}$ and the outer radius $r_{k}^{e}$. It is surrounded by insulation part with thickness $r_{k}^{e}-r_{k}^{i}$ and boundaries $\Gamma_{k}^{i}$ and $\Gamma_{k}^{e}$.

The multi-cable $M C=\left(\left(x_{0}, y_{0}, r_{0}^{i}, r_{0}^{e}\right), C_{1} \ldots C_{N}\right)$ consists of $\mathrm{N}$ single cables, has the center coordinates $\left(x_{0}, y_{0}\right)$, the inner radius $r_{0}^{i}$ and the outer radius $r_{0}^{e}$. It is surrounded by

*Corresponding author. Email addresses: zakaria.belhachmi@uha.fr (Z. Belhachmi), belhassen.meftahi@ enit.utm.tn (B. Meftahi), houcine.meftahi@enit.utm.tn (H. Meftahi) 
insulation layer with thickness $r_{0}^{e}-r_{0}^{i}$ and conductivity $\sigma^{e}$. Each single cable consists of a core part $\Omega_{k}^{\text {core }}$ with heat conductivity $\sigma_{k}^{\text {core }}$, carrying the current $I_{k}$, and an insulation part $\Omega_{k}^{i s o}$ with heat conductivity $\sigma_{k}^{i s o}$. The gaps between the single cables and the exterior insulation can be of solid material or air. They are modeled by pure conduction with heat conductivity $\sigma^{\text {gap }}$.

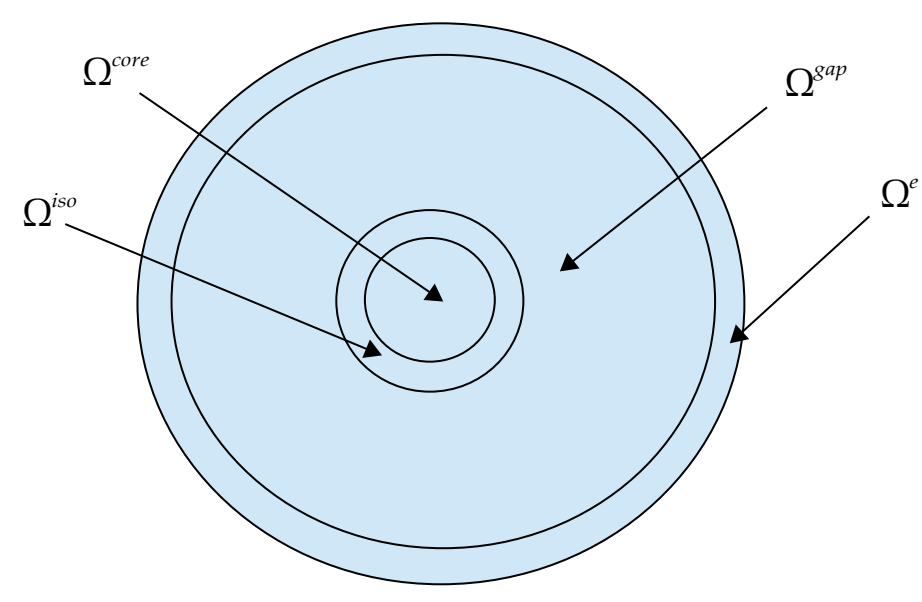

Figure 1: The cross section of a single cable.

The temperature distribution is described by the following Helmoltz equation :

$$
\begin{cases}-\operatorname{div}(\sigma \nabla u)-c u=f & \text { in } \Omega \backslash \Gamma^{i}, \\ \sigma_{e} \partial_{n} u+\alpha(u)\left(u-u_{a m b}\right)=0 & \text { on } \Gamma^{e}, \\ \llbracket u \rrbracket=0 & \text { on } \Gamma^{i}, \\ \llbracket \sigma \partial_{n} u \rrbracket=0 & \text { on } \Gamma^{i},\end{cases}
$$

where $\Omega=\cup_{k=1}^{N}\left(\Omega_{k}^{\text {core }} \cup \Omega_{k}^{i s o}\right) \cup \Omega^{\text {gap }} \cup \Omega^{e}$ is the two-dimensional cross section of the multicable with regular exterior boundary $\partial \Omega=\Gamma^{e}$ and interface boundaries $\Gamma^{i}=\bigcup_{k=1}^{N}\left(\Gamma_{k}^{e} \cup \Gamma_{k}^{i}\right) \cup$ $\Gamma^{g i}, \Gamma^{g i}$ represents the interface between the exterior insulation and the gaps, $\Gamma_{k}^{i}=\partial \Omega_{k}^{c o r e}$ and $\Gamma_{k}^{e}$ represents the external boundary of $\Omega_{k}^{i s o}$.

The heat conductivity $\sigma$, the linear temperature coefficient $c$ and the source term $f$ are given respectively by

$$
\begin{aligned}
\sigma & :=\sum_{k=1}^{N}\left(\sigma_{k}^{\text {core }} \mathbb{1}_{\Omega_{k}^{\text {core }}}+\sigma_{k}^{i s o} \mathbb{1}_{\Omega_{k}^{\text {iso }}}\right)+\sigma^{e} \mathbb{1}_{\Omega^{e}}+\sigma^{\text {gap }} \mathbb{1}_{\Omega^{\text {gap }}}, \\
c: & =\sum_{k=1}^{N} \frac{1}{n_{k}}\left(\frac{4 I_{k}}{d_{k}^{i n} \delta_{k} \pi}\right)^{2} \rho_{0, k} \alpha_{\rho, k} \mathbb{1}_{\Omega_{k}^{\text {core }}},
\end{aligned}
$$




$$
f:=\sum_{k=1}^{N} \frac{1}{n_{k}}\left(\frac{4 I_{k}}{d_{k}^{\text {in }} \delta_{k} \pi}\right)^{2} \rho_{0, k}\left(1-\alpha_{\rho, k} u_{r e f}\right) \mathbb{1}_{\Omega_{k}^{\text {core }}},
$$

where $d_{k}^{\text {in }}$ is the interior diameter of the k-th single cable, $n_{k}$ is the number of metallic conductor and $\delta_{k}$ is their diameter, $\rho_{0, k}$ is the electrical resistivity of $\Omega_{k}^{c o r e}$ at reference temperature $u_{r e f}$ (normally $20^{\circ} \mathrm{C}$ ), $u_{a m b}$ is the ambiant temperature and $\alpha_{\rho, k}$ the linear temperature coefficient of electrical resistivity. The heat transfer coefficient $\alpha(u)$ is nonlinearly temperature and geometry dependent quantity, incorporating the effects of radiation and convection at the transition from solid material to air.

$$
\alpha(u)=\alpha_{\text {conv }}(u)+\alpha_{\text {rad }}(u),
$$

where $\alpha_{\text {conv }}(u)$ is the convective part of $\alpha(u)$, summarizes the fluid dynamic properties of air and $\alpha_{\text {rad }}(u)$ is the radiative part, it is derived from the Stefan-Boltzmann law.

In the particular case of cylindrical multi-cable, the transfert coefficient $\alpha$ is given by

$$
\alpha(u)= \begin{cases}\alpha\left(u_{a \operatorname{mb}}\right) & \text { if } u<u_{a \operatorname{mb}} \\ \alpha(u) & \text { if } u_{\text {amb }} \leq u \leq u_{\max } \\ \alpha\left(u_{\max }\right) & \text { if } u>u_{\max }\end{cases}
$$

where

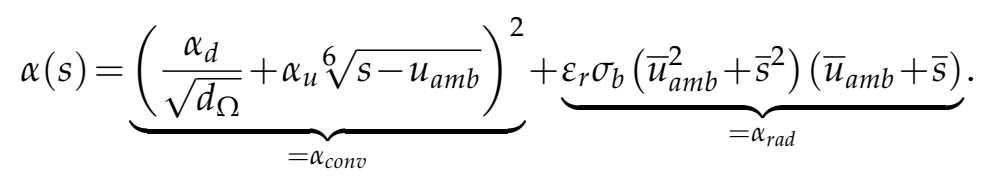

The parameters $\alpha_{d}$ and $\alpha_{u}$ describe the dependence of the convection term on the exterior multicable diameter $d_{\Omega}$ and the difference in temperature, respectively [11]. $\varepsilon_{r}$ is the emission coefficient of the conductor surface, $\sigma_{b}$ the Stefan-Boltzmann constant $(=5.67 e-$ 8 ) and $\bar{s}=u_{0}+s$ where $u_{0} \approx 273.15 \mathrm{~K}$ denotes the difference from $0^{\circ} \mathrm{C}$ to absolute zero. The maximal temperature satisfies $u_{\max } \leq 200^{\circ} \mathrm{C}$.

In this paper, we aim to find the best locations of electrical multicables in order to get suitable thermal environment. We mention that this problem was considered in [9]. For the numerical computation the authors develop an algorithm which is combination of a squeezing algorithm, helping to find feasible cable configurations for a genetic algorithm and a gradient-based shape optimization approach.

In this work, we use a topological and shape gradient strategy $[1,3,4,13,15-17,19]$ to find a desired multicable configuration.

The papaer is organized as follows in Section 2 we prove the existence and uniqueness of the direct problem and we introduce the minimization problem. In Section 3, we compute the shape derivative of the proposed cost functional using the framework of the min-sup differentiability. In Section 4 we give the asymptotic expansion of the cost functional. In the last section, we propose a numerical algorithm and we present some numerical simulations. 


\section{Existence and uniqueness for the Helmholtz equation}

In this section we prove the existence and uniqueness for the Helmholtz equation (1.1). For simplicity of calculus, we assume that $u_{a m b}=0$.

The weak formulation of problem (1.1) is given by:

Find $u \in H^{1}(\Omega)$ such that

$$
\int_{\Omega} \sigma \nabla u \cdot \nabla v d x-\int_{\Omega} c u v d x+\int_{\Gamma^{e}} \alpha(u) u v d s=\int_{\Omega} f v d s, \quad \forall v \in H^{1}(\Omega) .
$$

By a solution of problem (1.1) we actually mean a solution of (2.1). Throughout the rest of the paper, we assume that the fonctions $\alpha$ and $c$ satisfy the following hypothesis:

$\left(H_{1}\right)$ :

i) There exist constants $\alpha_{-}, \alpha_{+}$such that $0<\alpha_{-} \leq \alpha(x) \leq \alpha_{+}$,

ii) $\alpha$ is continuously differentiable on $(0, \infty)$,

iii) $\exists m>0$ such that $(\alpha(s) s)^{\prime}=\alpha(s)+\alpha^{\prime}(s) s \geq m$,

$\left(H_{2}\right)$ : There exist a positive constants $c_{+}, c_{-}$small enough such that $c_{-} \leq c \leq c_{+}<\alpha_{-}$.

Remark 2.1. The condition iii) imply that the function $s \rightarrow \alpha(s) s$ is strongly monotone with monotonicity constant $m$ :

$$
(\alpha(s) s-\alpha(t) t)(s-t) \geq m(s-t)^{2} .
$$

Theorem 2.1. Under hyporthesis $\left(H_{1}\right),\left(H_{2}\right)$, problem (2.1) has a unique solution $u \in H^{1}(\Omega)$.

Proof. Introduce the functional

$$
J(u)=\frac{1}{2} \int_{\Omega} \sigma|\nabla u|^{2}-c u^{2} d x+\int_{\Gamma^{e}} \int_{0}^{u} \alpha(t) t d t-\int_{\Omega} f u d x .
$$

It is easy to check that the functional $J$ is of classe $C^{1}$ on $H^{1}(\Omega)$. Its derivative can be computed as

$$
J^{\prime}(u) v=\int_{\Omega} \sigma \nabla u \cdot \nabla v d x-\int_{\Omega} c u v d x+\int_{\Gamma^{e}} \alpha(u) u v d s-\int_{\Omega} f v d s,
$$

and the first-order optimality condition

$$
J^{\prime}(u) v=0 \quad \forall v \in H^{1}(\Omega),
$$

implies that a minimizer of $J$ is a solution of (2.1).

To prove the existence of a weak solution it suffices to prove that $J$ is coercive. 
Denote

$$
\bar{u}:=\frac{1}{|\Omega|} \int_{\Omega} u d x \quad \text { the mean-value of } \mathrm{u} .
$$

Using Young-inequality, we obtain

$$
\begin{aligned}
J(u) & \geq \int_{\Omega} \sigma|\nabla u|^{2} d x-\int_{\Omega} c u^{2} d x-\frac{\epsilon}{2} \int_{\Omega}|u|^{2} d x-\frac{1}{2 \epsilon} \int_{\Omega}|f|^{2} d x+\frac{\alpha_{-}}{2} \int_{\Gamma^{e}} u^{2} d s \\
& \geq \min \left(\min (\sigma), \frac{\alpha_{-}}{2}\right)\left(\int_{\Omega}|\nabla u|^{2} d x+\int_{\Gamma^{e}} u^{2} d s\right)-\left(c_{+}+\frac{\epsilon}{2}\right) \int_{\Omega} u^{2} d x-\frac{1}{2 \epsilon} \int_{\Omega}|f|^{2} d x \\
& =\min \left(\min (\sigma), \frac{\alpha_{-}}{2}\right)\|u\|_{\star}^{2}-\left(c_{+}+\frac{\epsilon}{2}\right)\|u\|_{L^{2}(\Omega)}^{2}-\frac{1}{2 \epsilon} \int_{\Omega}|f|^{2} d x,
\end{aligned}
$$

where

$$
\|u\|_{\star}=\left(\int_{\Omega}|\nabla u|^{2} d x+\int_{\Gamma^{e}} u^{2} d s\right)^{1 / 2},
$$

is a norm equivalent to the natural norm of $H^{1}(\Omega)$. i.e.,

$$
c_{1}\|u\|_{H^{1}(\Omega)} \leq\|u\|_{\star} \leq c_{2}\|u\|_{H^{1}(\Omega)} .
$$

Using the fact that $\|u\|_{L^{2}(\Omega)}^{2} \leq\|u\|_{H^{1}(\Omega)}^{2}$, we obtain

$$
J(u) \geq\left(c_{1}^{2} \min \left(\min (\sigma), \frac{\alpha_{-}}{2}\right)-\left(c_{+}+\frac{\epsilon}{2}\right)\right)\|u\|_{H^{1}(\Omega)}^{2}-\frac{1}{2 \epsilon} \int_{\Omega}|f|^{2} d x .
$$

From hypothesis $\left(\mathrm{H}_{2}\right)$ and choosing $\epsilon$ small enough, we can conclude that

$$
\lim _{\|u\|_{H^{1}(\Omega)} \rightarrow \infty} J(u)=+\infty .
$$

In the next step we show that $J$ is strictly convex. We have

$$
\begin{aligned}
& \left(J^{\prime}(u)-J^{\prime}(v), u-v\right) \\
= & \int_{\Omega} \sigma|\nabla(u-v)|^{2} d x-\int_{\Omega} c(u-v)^{2} d x+\int_{\Gamma^{e}}(\alpha(u) u-\alpha(v) v)(u-v) d s .
\end{aligned}
$$

Using hypothesis $\left(H_{1}\right)$ and $\left(H_{2}\right)$, we can show that

$$
\left(J^{\prime}(u)-J^{\prime}(v), u-v\right) \geq 0,
$$

and if $\left(J^{\prime}(u)-J^{\prime}(v), u-v\right)=0$ then $u=v$. This concludes the proof.

In order to get a suitable thermal environment inside the multi-cable, we consider the following minimization problem:

$$
\text { minimize } J_{p}(\omega, u):=\frac{1}{p} \int_{\Omega}|u|^{p} d x, p \geq 2,
$$

subject to $\omega \in \mathcal{O}_{a d}$ and $u$ the solution of (1.1), 
where

$$
\mathcal{O}_{a d}:=\left\{\omega=\cup_{k=1}^{N} \omega_{k} \subset \Omega \backslash \Omega^{e}, \quad \omega_{k}=\Omega_{k}^{\text {core }} \cup \Omega_{k}^{i s o}, \quad \Omega_{k}^{\text {core }} \text { is surrounded by } \Omega_{k}^{\text {iso }}\right\} .
$$

Throughout this paper, we assume that all interface boundaries $\Gamma_{k}^{i}=\partial \Omega_{k}^{i s o}, \Gamma_{k}^{e}=$ the external boundary of $\Omega^{i s o}, \Gamma^{g i}=$ the internal boundary of $\Omega^{e}$ and $\Gamma^{e}=$ the external boundary of $\Omega^{e}$ are $C^{2}$-smooth.

The numerical resolution of (2.2) requires the sensitivity analysis of $J_{p}$ with respect to $\omega$. The differentiation with respect to the shape $\omega$ is the main purpose of the following section.

\section{Shape derivative}

\subsection{Preliminaries}

In this section we recall some basic facts about the velocity method from shape optimization used to calculate the shape derivatives of the functional $J_{p}$; see $[7,18]$. In the velocity (or speed) method a domain $\Omega$ is deformed by the action of a velocity field $V$. The evolution of the domain is described by the following dynamical system:

$$
\left\{\begin{array}{l}
\frac{d}{d t} x(t)=V(x(t)), t \in[0, \varepsilon), \\
x(0)=X
\end{array}\right.
$$

for some real number $\varepsilon>0$. Assume $V \in \mathcal{D}^{1}\left(\Omega ; \mathbb{R}^{2}\right)$ where $\mathcal{D}^{1}\left(\Omega ; \mathbb{R}^{2}\right)$ denotes the space of continuously differentiable functions with compact support in $\Omega$, then the ordinary differential equation (3.1) has a unique solution. This allows us to define the diffeomorphism

$$
T_{t}: \mathbb{R}^{2} \rightarrow \mathbb{R}^{2}: X \mapsto T_{t}(X):=x(t) .
$$

For $t \in[0, \varepsilon), T_{t}$ is invertible. Furthermore, the Jacobian $\xi(t)$ is strictly positive

$$
\forall t \in[0, \varepsilon), \quad \xi(t)=\left|\operatorname{det} D T_{t}(X)\right|>0,
$$

where $D T_{t}(X)$ is the Jacobian matrix of the transformation $T_{t}$ associated with the velocity field $V$. In the sequel, we use the following notation : $M^{-1}$ for the inverse of $M$ and $M^{-*}$ for the transpose of its inverse. We also denote by

$$
w(t)=\xi(t)\left|\left(D T_{t}\right)^{-*} n\right|,
$$

the tangential Jacobian of $T_{t}$ on $\partial \Omega$.

Remark 3.1. As the sets $\omega_{k}$ are made of two pieces $\left(\Omega_{k}^{\text {core }}\right.$ and $\left.\Omega_{k}^{i s o}\right)$, we will restrict the fields $V$ to those preserving such structure. This easily done for example if we concatenate local smooth fields. 
Proposition 3.1 ([7,18]). For a function $\varphi \in W_{\text {loc }}^{1,1}\left(\mathbb{R}^{2}\right)$ and $V \in \mathcal{D}^{1}\left(\mathbb{R}^{2}\right)$, we have the following formulae

$$
\begin{aligned}
& \nabla\left(\varphi \circ T_{t}\right)=D T_{t}^{*}(\nabla \varphi) \circ T_{t}, \\
& \frac{d}{d t}\left(\varphi \circ T_{t}\right)=(\nabla \varphi \cdot V) \circ T_{t}, \\
& \frac{d \xi(t)}{d t}=[\operatorname{div} V] \circ T_{t} \xi(t), \\
& w^{\prime}(0)=\operatorname{div}(V)-D V n \cdot n .
\end{aligned}
$$

Let $J$ be a real valued function $J: \Omega \longrightarrow \mathbb{R}$. We say that $J$ has a Eulerian semiderivative at $\Omega$ in the direction $V$ if the following limit exists and is finite:

$$
d J(\Omega ; V)=\lim _{t \searrow 0} \frac{J\left(T_{t}(\Omega)\right)-J(\Omega)}{t} .
$$

If $V \longrightarrow d J(\Omega ; V))$ is linear and continuous, we say that $J$ is shape differentiable at $\Omega$.

Definition $3.1([7,18])$. Let $\Omega$ be an open domain of class $C^{2}$ with compact boundary $\partial \Omega$. We denote by $U(\partial \Omega)$ a neighborhood of $\partial \Omega$.

(i) Let $f \in C^{1}(\partial \Omega)$ and $\widetilde{f}$ be an extension of $f$ in a neighborhood of $\partial \Omega$. The tangential gradient of $f$ at a point of $\partial \Omega$ is defined as

$$
\nabla_{\tau} f=\nabla \widetilde{f}-\partial_{n} \tilde{f} n,
$$

where $n$ is the outward unit normal vector to $\partial \Omega$.

(ii) For a vector function $v \in C^{1}(\partial \Omega)^{d}, d \geq 1$ and its extension $\widetilde{v}$, the tangential divergence is defined as

$$
\operatorname{div}_{\tau} v=\operatorname{div}(\widetilde{v})-D \widetilde{v} n \cdot n,
$$

where D $\widetilde{v}$ denotes the Jacobian matrix of $\widetilde{v}$.

Note that the tangential divergence and gradient are independent of the extension.

\subsection{Min-sup formulation}

In what follows we focus on the computation of the shape derivative of $J_{p}$. We introduce the Lagrangian functional

$$
G(\omega, \varphi, \psi)=J_{p}(\omega, \varphi)+b(\omega, \varphi, \psi)-l(\psi), \quad \forall \varphi, \psi \in H^{1}(\Omega),
$$

where

$$
b(\omega, \varphi, \psi)=\int_{\Omega} \sigma \nabla \varphi \cdot \nabla \psi d x-\int_{\Omega} c \varphi \psi d x+\int_{\partial \Omega} \alpha(\varphi) \varphi \psi d s, \quad l(\psi)=\int_{\Omega} f \psi d s .
$$


Then, it is easy to check that

$$
J_{p}(\omega, u(\omega))=\min _{\varphi \in H^{1}(\Omega)} \sup _{\psi \in H^{1}(\Omega)} G(\omega, \varphi, \psi),
$$

since

$$
\sup _{\psi \in H^{1}(\Omega)} G(\omega, \varphi, \psi)=\left\{\begin{array}{cl}
J_{0}(\omega, u(\omega)) & \text { if } \varphi=u(\omega), \\
+\infty & \text { otherwise. }
\end{array}\right.
$$

It is easily shown that the functional $G$ is convex continuously differentiable with respect to $\varphi$ and concave continuously differentiable with respect to $\psi$. Therefore, according to Ekeland and Temam [8], the functional $G$ has a saddle point $(u, v)$ if and only if $(u, v)$ solves the following system:

$$
\begin{aligned}
& \partial_{\psi} G(\omega, u, v ; \hat{\psi})=\partial_{\psi} b(\omega, u, v ; \hat{\psi})-\partial_{\psi} l(v ; \hat{\psi})=0, \\
& \partial_{\varphi} G(\omega, u, v ; \hat{\varphi})=\partial_{\varphi} J_{p}(\omega, u ; \hat{\varphi})+\partial_{\varphi} b(\omega, u, v ; \hat{\varphi})=0,
\end{aligned}
$$

for all $\hat{\psi} \in H^{1}(\Omega)$ and $\hat{\varphi} \in H^{1}(\Omega)$. This yields that $G$ has a saddle point $(u, v)$, where the state $u$ is the unique solution of (1.1) and the adjoint state $v=v(\omega)$ is the solution of the following adjoint problem:

$$
\int_{\Omega} \sigma \nabla v \cdot \nabla \hat{v} d x-\int_{\Omega} c v \hat{v} d x+\int_{\Gamma^{e}}\left(\alpha(u)+\alpha^{\prime}(u) u\right) v \hat{v} d s+\int_{\Omega} u|u|^{p-2} \hat{v} d x=0,
$$

for all $\hat{v} \in H^{1}(\Omega)$.

Since $u$ is Hölder continuous (from elliptic regularity results), $u|u|^{p-2}$ is at least in $L^{2}(\Omega)$. Therefore problem (3.9) has unique solution $v \in H^{1}(\Omega)$. Summarizing the above, we have obtained

Theorem 3.1. The functional $J_{p}(\omega, u(\omega))$ is given by

$$
J_{p}(\omega, u(\omega))=\min _{\varphi \in H^{1}(\Omega)} \sup _{\psi \in H^{1}(\Omega)} G(\omega, \varphi, \psi),
$$

The unique saddle point for $G$ is given by $(u, v)$, where $u$ solves the direct problem (1.1) and $v$ solves the adjoint problem (3.9).

Similarly, the previous analysis holds for the functional depending on the transformed subdomain $\omega_{t}=T_{t}(\omega)$. Thus, we have

$$
J_{p}\left(\omega_{t}, u\left(\omega_{t}\right)=\min _{\varphi \in H^{1}(\Omega)} \sup _{\psi \in H^{1}(\Omega)} G\left(\omega_{t}, \varphi, \psi\right) .\right.
$$

The corresponding saddle point $\left(u\left(\omega_{t}\right), v\left(\omega_{t}\right)\right)$ is characterized by

$$
\begin{array}{ll}
\partial_{\psi} G\left(\omega_{t}, u\left(\omega_{t}\right), v\left(\omega_{t}\right) ; \hat{\psi}\right)=0, & \forall \hat{\psi} \in H^{1}(\Omega), \\
\partial_{\varphi} G\left(\omega_{t}, u\left(\omega_{t}\right), v\left(\omega_{t}\right) ; \hat{\varphi}\right)=0, & \forall \hat{\varphi} \in H^{1}(\Omega) .
\end{array}
$$




\subsection{Shape derivative by the min-sup differentiability}

In this section we apply Theorem 6.1 to compute the shape derivative of $J_{p}$. Let us consider transformation $T_{t}$ defined by (3.2) with $V \in \mathcal{D}^{1}\left(\Omega, \mathbb{R}^{2}\right)$. In this case $T_{t}(\Omega)=\Omega$ but in general $T_{t}(\omega) \neq \omega$. Our aim is to compute the derivative of $J_{p}\left(\omega_{t}, u\left(\omega_{t}\right)\right)$ with respect to the parameter $t \geq 0$.

In order to differentiate $G\left(\omega_{t}, \varphi, \psi\right)$ with respect to $t$, the integrals in $G\left(\omega_{t}, \varphi, \psi\right)$ need to be transported back on the reference interface $\omega$ using the transformation $T_{t}$. However, composing by $T_{t}$ inside the integrals creates terms $\varphi \circ T_{t}$ and $\psi \circ T_{t}$ which might be non-differentiable. To avoid this problem, we need to parameterize the space $H^{1}(\Omega)$ by composing the elements of $H^{1}(\Omega)$ with $T_{t}^{-1}$. Following this argument, we rewrite (3.11) as

$$
J_{p}\left(\omega_{t}, u\left(\omega_{t}\right)\right)=\min _{\varphi \in H^{1}(\Omega)} \sup _{\psi \in H^{1}(\Omega)} \tilde{G}(t, \varphi, \psi),
$$

where

$$
\tilde{G}(t, \varphi, \psi):=G\left(\omega_{t}, \varphi \circ T_{t}^{-1}, \psi \circ T_{t}^{-1}\right) .
$$

Note that since $T_{t}(\Omega)=\Omega$ we have $H^{1}\left(T_{t}(\Omega)\right)=H^{1}(\Omega)$ and the sets over which the minimum and supremum are taken in (3.12) stay unchanged. Furthermore $\left(u^{t}, v^{t}\right)=$ $\left(u\left(\omega_{t}\right) \circ T_{t}, v\left(\omega_{t}\right) \circ T_{t}\right)$ is the saddle point of $\tilde{G}$.

We can rewrite expression (3.13) on the fixed domain $\Omega$ by using the transformation $T_{t}$. This yields

$$
\begin{gathered}
\tilde{G}(t, \varphi, \psi)=\frac{1}{p} \int_{\Omega}|\varphi|^{p} \xi(t) d x+\int_{\Omega} \sigma A(t) \nabla \varphi \cdot \nabla \psi d x-\int_{\Omega} c \varphi \psi \xi(t) d x \\
-\int_{\Omega} f \circ T_{t} \psi \xi(t) d x+\int_{\partial \Omega} w(t) \alpha(\varphi) \varphi \psi d s,
\end{gathered}
$$

where

$$
A(t):=\left(D T_{t}\right)^{-*}\left(D T_{t}\right)^{-1} \xi(t),
$$

and $\xi(t), \omega(t)$ are defined in (3.3),(3.4), respectively. The saddle point $\left(u^{t}, v^{t}\right)$ is characterized by

$$
\begin{aligned}
& \int_{\Omega} \sigma A(t) \nabla u^{t} \cdot \nabla \psi d x-\int_{\Omega} c u^{t} \psi \xi(t) d x+\int_{\partial \Omega} w(t) \alpha\left(u^{t}\right) u^{t} \psi d s=\int_{\Omega} \xi(t) f \circ T_{t} \psi d s, \\
& \int_{\Omega} \sigma A(t) \nabla v^{t} \cdot \nabla \varphi d x-\int_{\Omega} c v^{t} \varphi d x+\int_{\partial \Omega} w(t)\left(\alpha^{\prime}\left(u^{t}\right)+\alpha\left(u^{t}\right)\right) v^{t} \varphi d s \\
= & -\int_{\Omega} \xi(t) u^{t}\left|u^{t}\right|^{p-2} \varphi d x,
\end{aligned}
$$

for all $\psi \in H^{1}(\Omega)$ and $\varphi \in H^{1}(\Omega)$.

Now we are ready to compute the limit

$$
d J_{p}(\omega ; V):=\lim _{t \rightarrow 0} \frac{\mathcal{J}_{p}\left(\omega_{t}\right)-\mathcal{J}_{p}(\omega)}{t}
$$


where

$$
\mathcal{J}_{p}\left(\omega_{t}\right):=J_{p}\left(\omega_{t}, u\left(\omega_{t}\right)\right) .
$$

Theorem 3.2. The functional $J_{p}$ is shape differentiable and its shape derivative in the direction $V \in \mathcal{D}^{1}(\Omega)$ is given by

$$
\begin{gathered}
d J_{p}(\omega, V)=\frac{1}{p} \int_{\Omega}|u|^{p} \operatorname{div} V d x+\int_{\Omega} \sigma\left(\operatorname{div} V-D V^{T}-D V\right) \nabla u \cdot \nabla v d x \\
-\int_{\Omega} c u v \operatorname{div} V d x-\int_{\Omega} f v \operatorname{div} V d x,
\end{gathered}
$$

where $u$ is solution of the problem (1.1) and the adjoint state $v$ is solution of

$$
\int_{\Omega} \sigma \nabla v \cdot \nabla \hat{v} d x-\int_{\Omega} c v \hat{v} d x+\int_{\partial \Omega}\left(\alpha(u)+\alpha^{\prime}(u) u\right) v \hat{v} d s+\int_{\Omega} u|u|^{p-2} \hat{v} d x=0,
$$

for all $\hat{v} \in H^{1}(\Omega)$.

Proof. We apply Theorem 6.1 given in the appendix to compute the shape derivative of $\mathcal{J}_{p}$; see Section 6.1. To this end, we should verify the four assumptions $\left(H_{1}\right)-\left(H_{4}\right)$.

Assumption $\left(H_{1}\right)$ : Given $(\beta, \gamma)$ satisfying $0<c_{+}<\beta<\gamma$, we can find $\varepsilon>0$ such that

$$
\forall \eta \in \mathbb{R}^{2}, \quad \beta|\eta|^{2} \leq \sigma A(t) \eta \cdot \eta \leq \gamma|\eta|^{2}, \text { for } t \in[0, \varepsilon] .
$$

As in Theorem 6.1, introduce the sets

$$
\begin{aligned}
& X(t):=\left\{x^{t} \in H^{1}(\Omega): \sup _{y \in H^{1}(\Omega)} \tilde{G}\left(t, x^{t}, y\right)=\inf _{x \in H^{1}(\Omega)} \sup _{y \in H^{1}(\Omega)} \tilde{G}(t, x, y)\right\}, \\
& Y(t):=\left\{y^{t} \in H^{1}(\Omega): \inf _{x \in H^{1}(\Omega)} \tilde{G}\left(t, x, y^{t}\right)=\sup _{y \in H^{1}(\Omega)} \inf _{x \in H^{1}(\Omega)} \tilde{G}(t, x, y)\right\} .
\end{aligned}
$$

We obtain

$$
\forall t \in[0, \varepsilon] \quad S(t)=X(t) \times Y(t)=\left\{u^{t}, v^{t}\right\} \neq \varnothing,
$$

and assumption $\left(H_{1}\right)$ is satisfied.

Assumption $\left(H_{2}\right)$ : Defining $B(t)=D T_{t}^{-*}$, we may compute

$$
\begin{aligned}
& B^{\prime}(t)=-B(t) D V^{*} B(t), \quad \xi^{\prime}(t)=\operatorname{tr}\left(D V B(t)^{*}\right) \xi(t), \\
& A^{\prime}(t)=-A(t) \operatorname{tr}\left(D V B(t)^{*}\right)+A(t) B(t)^{*} D V+D V^{*} B(t) A(t), \\
& w^{\prime}(t)=\xi^{\prime}(t)|B(t) n|+\xi(t)|B(t) n|^{-1} B^{\prime}(t) n .
\end{aligned}
$$

Consequently we obtain the derivatives

$$
\begin{gathered}
\partial_{t} \tilde{G}(t, \varphi, \psi)=\frac{1}{p} \int_{\Omega}|\varphi|^{p} \xi^{\prime}(t) d x+\int_{\Omega} \sigma A^{\prime}(t) \nabla \varphi \cdot \nabla \psi d x-\int_{\Omega} c \varphi \psi \xi^{\prime}(t) d x \\
+\int_{\partial \Omega} w^{\prime}(t) \alpha(\varphi) \varphi \psi d s-\int_{\Omega} f \circ T_{t} \xi^{\prime}(t) d x .
\end{gathered}
$$


At $t=0$ we also have

$$
A^{\prime}(0)=\operatorname{div}(V) I_{2}-D V^{*}-D V, \quad w^{\prime}(0)=\operatorname{div}_{\tau} V,
$$

where $I_{2}$ is the $2 \times 2$ identity matrix. Since $V \in \mathcal{D}^{1}(\Omega), t \rightarrow D T_{t}$ is continuous in $[0, \varepsilon]$ and consequently also $t \mapsto\left(B(t), A(t), A^{\prime}(t), w(t), w^{\prime}(t)\right)$. Therefore the partial derivatives $\partial_{t} \tilde{G}(t, \varphi, \psi)$ exists everywhere in $[0, \varepsilon]$ and the condition $\left(H_{2}\right)$ is satisfied.

Assumptions $\left(H_{3}\right)$ and $\left(H_{4}\right)$ : We show first the boundedness of $\left(u^{t}, v^{t}\right)$. Let $\psi=u^{t}$ in the variational equation (3.15). By the choice of $\varepsilon$ satisfying the condition (3.19), with

$$
T_{t}(x)=x, \quad \xi(t)=1 \quad w(t)=1 \text { on } \partial \Omega,
$$

and hypotheses $H_{1}$ and $H_{2}$ in section 2, we get from (3.15)

$$
\beta\left\|\nabla u^{t}\right\|_{L^{2}(\Omega)}^{2}-c_{+}\left\|u^{t}\right\|_{L^{2}(\Omega)}^{2}+\alpha_{-}\left\|u^{t}\right\|_{L^{2}(\partial \Omega)}^{2} \leq\left\|\xi(t) f \circ T_{t}\right\|_{L^{2}(\Omega)}\left\|u^{t}\right\|_{L^{2}(\Omega)} .
$$

Using Young's inequality, we obtain

$$
\beta\left\|\nabla u^{t}\right\|_{L^{2}(\Omega)}^{2}-c_{+}\left\|u^{t}\right\|_{L^{2}(\Omega)}^{2}+\alpha_{-}\left\|u^{t}\right\|_{L^{2}(\partial \Omega)}^{2} \leq \frac{1}{2 r}\left\|\xi(t) f \circ T_{t}\right\|_{L^{2}(\Omega)}^{2}+\frac{r}{2}\left\|u^{t}\right\|_{L^{2}(\Omega)}^{2},
$$

for some $r>0$. This imply that

$$
\beta\left\|\nabla u^{t}\right\|_{L^{2}(\Omega)}^{2}-\left(c_{+}+\frac{r}{2}\right)\left\|u^{t}\right\|_{L^{2}(\Omega)}^{2}+\left(\alpha_{-}\right)\left\|u^{t}\right\|_{L^{2}(\partial \Omega)}^{2} \leq \frac{1}{2 r}\left\|\xi(t) f \circ T_{t}\right\|_{L^{2}(\Omega)}^{2} .
$$

We choose $s$ such that $\alpha>s / 2$, and using the fact that

$$
\|u\|^{2}:=\|\nabla u\|_{L^{2}(\Omega)}^{2}+\|u\|_{L^{2}(\partial \Omega)}^{2}
$$

is a norm on $H^{1}(\Omega)$ equivalent to the natural norm (cf. [14]), we obtain

$$
\min \left(\beta, \alpha_{-}\right)\left\|u^{t}\right\|_{H^{1}(\Omega)}^{2}-\left(c_{+}+\frac{r}{2}\right)\left\|u^{t}\right\|_{L^{2}(\Omega)}^{2} \leq \frac{1}{2 r}\left\|\xi(t) f \circ T_{t}\right\|_{L^{2}(\Omega)}^{2} .
$$

Choosing $r$ such that $\min \left(\beta, \alpha_{-}\right)-\left(c_{+}+r / 2\right)>0$, we deduce that

$$
\left\|u^{t}\right\|_{H^{1}(\Omega)}^{2} \leq C\left(\frac{1}{2 r}\left\|\xi(t) f \circ T_{t}\right\|_{L^{2}(\Omega)}^{2}\right),
$$

where $C$ is a positive constant. Since $\xi(t) \rightarrow 1$ as $t \rightarrow 0$ and $f \circ T_{t} \rightarrow f$ in $L^{2}(\Omega)$ (see [7, Lemma $2.1 \mathrm{pp}$ 397]), then $u^{t}$ is bounded:

$$
\text { there exists } c>0 \text { such that } \sup _{t \in[0, \epsilon]}\left\|u^{t}\right\|_{H^{1}(\Omega)} \leq c \text {. }
$$


We apply the same technique to the variational equation (3.16) and we are able to show that the function $v^{t}$ is bounded. The next step is to show the continuity with respect to $t$ of the vector $\left(u^{t}, v^{t}\right)$. Subtracting (3.15) at $t>0$ and $t=0$ and choosing $\psi=u-u^{t}$ yields

$$
\begin{aligned}
& \int_{\Omega} \sigma\left|\nabla\left(u-u^{t}\right)\right|^{2} d x-\int_{\Omega} c\left|u-u^{t}\right|^{2} d x+\int_{\partial \Omega} \alpha\left(u^{t}\right)\left|u-u^{t}\right|^{2} d s \\
& =\int_{\Omega}\left(\sigma A(t)-\sigma I_{2}\right) \nabla u^{t} \cdot \nabla\left(u-u^{t}\right) d x-\int_{\Omega}(\xi(t)-1) c u^{t}\left(u-u^{t}\right) d x \\
& \quad+\int_{\partial \Omega}\left(\xi(t) \alpha\left(u^{t}\right)-\alpha(u)\right) u^{t}\left(u-u^{t}\right) d s+\int_{\Omega}\left(\xi(t) f \circ T_{t}-f\right)\left(u-u^{t}\right) d x \\
& \leq\left\|\sigma A(t)-\sigma I_{2}\right\|_{L^{\infty}(\Omega)}\left\|\nabla u^{t}\right\|_{L^{2}(\Omega)}\left\|\nabla\left(u-u^{t}\right)\right\|_{L^{2}(\Omega)} \\
& \quad+c_{+}\|(\xi(t)-1)\|_{L^{\infty}(\Omega)}\left\|u^{t}\right\|_{L^{2}(\Omega)}\left\|u-u^{t}\right\|_{L^{2}(\Omega)} \\
& \quad+\left\|\left(\xi(t) \alpha\left(u^{t}\right)-\alpha(u)\right)\right\|_{L^{\infty}(\partial \Omega)}\left\|u^{t}\right\|_{L^{2}(\partial \Omega)}\left\|u-u^{t}\right\|_{L^{2}(\partial \Omega)} \\
& \quad+\left\|\xi(t) f \circ T_{t}-f\right\|_{L^{2}(\Omega)}\left\|u-u^{t}\right\|_{L^{2}(\Omega)} .
\end{aligned}
$$

Furthermore due to the boundedness of $u^{t}$ and the fact that $\|u\|^{2}:=\|\nabla u\|_{L^{2}(\Omega)}^{2}+\|u\|_{L^{2}(\partial \Omega)}^{2}$ is a norm on $H^{1}(\Omega)$ equivalent to the natural norm, we obtain

$$
\begin{aligned}
\left\|u^{t}-u\right\|_{H^{1}(\Omega)} \leq c & \left(\left\|\sigma A(t)-\sigma I_{2}\right\|_{L^{\infty}(\Omega)}+\|\xi(t)-1\|_{L^{\infty}(\Omega)}\right. \\
& \left.+\left\|\xi(t) \alpha\left(u^{t}\right)-\alpha(u)\right\|_{L^{\infty}(\partial \Omega)}+\left\|\xi(t) f \circ T_{t}-f\right\|_{L^{2}(\Omega)}\right) .
\end{aligned}
$$

Due to the strong continuity of $A(t)$ (as a function of $t$ ) and $\xi(t) f \circ T_{t} \rightarrow f, \xi(t) \alpha\left(u^{t}\right) \rightarrow \alpha(u)$ in $L^{2}(\Omega)$ as $t \rightarrow 0$, one deduces that $u^{t} \rightarrow u$ in $H^{1}(\Omega)$ as $t \rightarrow 0$. Concerning the continuity of $v^{t}$, one may show from (3.16) that $v^{t} \rightarrow v$ in $H^{1}(\Omega)$. Finally in view of the strong continuity of

$$
(t, \varphi) \rightarrow \partial_{t} \tilde{G}(t, \varphi, \psi) \text { and }(t, \psi) \rightarrow \partial_{t} \tilde{G}(t, \varphi, \psi),
$$

assumptions $\left(H_{3}\right)$ and $\left(H_{4}\right)$ are verified. All assumptions of Theorem 6.1 are satisfied and therefore, we obtain

$$
d \mathcal{J}_{p}(\omega ; V)=\left.\partial_{t} \tilde{G}(t, u, v)\right|_{t=0^{\prime}}
$$

where

$$
\begin{gathered}
\left.\partial_{t} \tilde{G}(t, u, v)\right|_{t=0}=\frac{1}{p} \int_{\Omega}|u|^{p} \operatorname{div}(V) d x+\int_{\Omega} \sigma A^{\prime}(0) \nabla u \cdot \nabla v d x \\
-\int_{\Omega} c u v \operatorname{div}(V) d x-\int_{\Omega} f \operatorname{div}(V) d x .
\end{gathered}
$$

This completes the proof. 
Theorem 3.3. (Boundary expression). The shape derivative of the functional $J_{p}$ in the direction $V \in \mathcal{D}^{1}(\Omega)$ is given by

$$
\begin{aligned}
D J_{p}(\omega, V)=- & \sum_{k=1}^{N} \int_{\Gamma_{k}^{i}} \llbracket \sigma \partial_{n} u \partial_{n} v \rrbracket_{\Gamma_{1}} V \cdot n d s-\sum_{k=1}^{N} \int_{\Gamma_{k}^{e}} \llbracket \sigma \partial_{n} u \partial_{n} v \rrbracket_{\Gamma_{2}} V \cdot n d s \\
& +\sum_{k=1}^{N} \int_{\Gamma_{k}^{i}} \llbracket \sigma \rrbracket_{\Gamma_{k}^{i}} \nabla_{\Gamma_{k}^{i}} u \cdot \nabla_{\Gamma_{k}^{i}} v d s+\sum_{k=1}^{N} \int_{\Gamma_{k}^{e}} \llbracket \sigma \rrbracket_{\Gamma_{k}^{e}} \nabla_{\Gamma_{k}^{e}} u \cdot \nabla_{\Gamma_{k}^{e}} v d s \\
& -\sum_{k=1}^{N} \int_{\Gamma_{k}^{i}} \llbracket c u v \rrbracket_{\Gamma_{k}^{i}} V \cdot n d s-\sum_{k=1}^{N} \int_{\Gamma_{k}^{e}} \llbracket c u v \rrbracket_{\Gamma_{k}^{e}} V \cdot n d s \\
& -\sum_{k=1}^{N} \int_{\Gamma_{k}^{i}} \llbracket f v \rrbracket_{\Gamma_{k}^{i}} V \cdot n d s-\sum_{k=1}^{N} \int_{\Gamma_{k}^{e}} \llbracket f v \rrbracket_{\Gamma_{k}^{e}} V \cdot n d s .
\end{aligned}
$$

Remark 3.2. Note that the volume expression of the shape derivative presented in Thorem 3.2 can be rewritten in canonical form as

$$
d \mathcal{J}_{p} J(\omega ; V)=\int_{\Omega} \mathrm{S}: D V d x
$$

where

$$
\mathrm{S}=-\sigma(\nabla u \otimes \nabla v+\nabla v \otimes \nabla u)+\sigma(\nabla u \cdot \nabla v) I+\frac{1}{p}|u|^{p} I-c u v I-f v I .
$$

Using standard tensor relation $(\nabla u \otimes \nabla) n=(\nabla v \cdot n) \nabla u$, one may obtain directly the boundary expression of the shape derivative presented in Theorem 3.3. For more details about tensor representation, we refer the reader to [10].

\section{Topological derivative}

In this section, we assume that $\Omega=\left(\Omega^{\text {core }} \cup \Omega^{\text {iso }}\right) \cup \Omega^{g a p} \cup \Omega^{e}$ (See, Figure 1) where $\Omega^{\text {core }}:=$ $x_{0}+\delta \varepsilon B$ and $\Omega^{i s}$ is such that $\Omega^{\text {core }} \cup \Omega^{i s o}=x_{0}+\varepsilon B$, where $B$ is the unit ball in $\mathbb{R}^{2}, \varepsilon>0$ and $0<\delta<1$. We rewrite $\Omega_{\varepsilon}^{\text {core }}$ and $\Omega_{\varepsilon}^{\text {iso }}$ instead of $\Omega^{\text {core }}$ and $\Omega^{i s o}$. This allows to perform an asymptotic expansion of the shape functional $J_{p}\left(\omega_{\varepsilon}\right)$ where $\omega_{\varepsilon}:=\Omega_{\varepsilon}^{\text {core }} \cup \Omega_{\varepsilon}^{\text {iso }}$. We also introduce $\Gamma_{\varepsilon}:=\Gamma_{\varepsilon}^{\text {core }} \cup \Gamma_{\mathcal{\varepsilon}}^{\text {iso }}$ where $\Gamma_{\varepsilon}^{\text {core }}:=\partial \Omega_{\varepsilon}^{\text {core }}$ and $\Gamma_{\mathcal{\varepsilon}}^{\text {iso }}$ the outer boundary of $\Omega_{\mathcal{\varepsilon}}^{\text {iso }}$.

In the perturbed domain, the state $u_{\varepsilon}$ is solution to the following problem :

$$
\begin{cases}-\operatorname{div}\left(\sigma_{\varepsilon} \nabla u_{\varepsilon}\right)-c_{\varepsilon} u_{\varepsilon}=f_{\varepsilon} & \text { in } \Omega \backslash \Gamma_{\varepsilon}, \\ \sigma_{e} \partial_{n} u_{\varepsilon}+\alpha\left(u_{\varepsilon}\right)\left(u_{\varepsilon}-u_{a m b}\right)=0 & \text { on } \Gamma^{e}, \\ \llbracket u_{\varepsilon} \rrbracket=0 & \text { on } \Gamma_{\varepsilon}, \\ \llbracket \sigma \partial_{n} u_{\varepsilon} \rrbracket=0 & \text { on } \Gamma_{\varepsilon},\end{cases}
$$


where

$$
\begin{aligned}
& \sigma_{\varepsilon}=\sigma^{\text {core }} \mathbb{1}_{\Omega_{\varepsilon}^{\text {core }}}+\sigma^{i s o} \mathbb{1}_{\Omega_{\varepsilon}^{i s o}}+\sigma^{e} \mathbb{1}_{\Omega^{e}}+\sigma^{\text {gaps }} \mathbb{1}_{\Omega \backslash \overline{\Omega_{\varepsilon}^{\text {core }} \cup \Omega_{\varepsilon}^{i s o} \cup \Omega^{e}},}, \\
& c_{\varepsilon}=c^{\text {core }} \mathbb{1}_{\Omega_{\varepsilon}^{\text {core }}}, \quad \text { and } \quad f_{\varepsilon}=f^{\text {core }} \mathbb{1}_{\Omega_{\varepsilon}^{\text {core }}} .
\end{aligned}
$$

The functions $f^{\text {core }}, c^{\text {core }}$ are defined in (1.3) and (1.4) . The variational formulation associated with the problem (4.1) is defined by :

$$
\text { find } u_{\varepsilon} \in H^{1}(\Omega) \text {, such that , } F_{\varepsilon}\left(u_{\varepsilon}, v\right)=0, \quad \forall v \in H^{1}(\Omega),
$$

where

$$
F_{\varepsilon}\left(u_{\varepsilon}, v\right)=\int_{\Omega} \sigma_{\varepsilon} \nabla u_{\varepsilon} \cdot \nabla v d x-\int_{\Omega} c_{\varepsilon} u_{\varepsilon} v d x+\int_{\partial \Omega} \alpha(u)\left(u-u_{a m b}\right) v d s-\int_{\Omega} f v d x .
$$

The asymptotic expansion of the cost functional will be provided by the following proposition

Proposition 4.1. Let $\mathcal{V}$ be a real Hilbert space. For $\varepsilon \in[0, \xi), \xi \geq 0$, we consider

- a differentiable map $F_{\varepsilon}: \mathcal{V} \rightarrow \mathcal{V}^{\prime}$,

- a function $u_{\varepsilon} \in \mathcal{V}$ satisfying

$$
F_{\varepsilon}\left(u_{\varepsilon}\right)=0,
$$

- a differentiable functional $J_{\varepsilon}: \mathcal{V} \longrightarrow \mathbb{R}$,

We assume that there exists $v_{0} \in \mathcal{V}$, called adjoint state, solving

$$
\left\langle D F_{0}\left(u_{0}\right) \varphi, v_{0}\right\rangle_{\mathcal{V}^{\prime}, \mathcal{V}}=-D J_{0}\left(u_{0}\right) \varphi, \quad \forall \varphi \in \mathcal{V} .
$$

Suppose that there exists real numbers $\delta F_{1}, \delta F_{2}, \delta J_{1}$ and $\delta J_{2}$ as well as a function $h(\varepsilon)$ tending to zero with $\varepsilon$ such that when $\varepsilon \rightarrow 0$

$$
\begin{aligned}
& \left\langle F_{\varepsilon}\left(u_{\varepsilon}\right)-F_{0}\left(u_{\varepsilon}\right), v_{0}\right\rangle=h(\varepsilon) \delta F_{1}+o(h(\varepsilon)), \\
& \left\langle F_{0}\left(u_{\varepsilon}\right)-F_{0}\left(u_{0}\right)-D F_{0}\left(u_{0}\right)\left(u_{\varepsilon}-u_{0}\right), v_{0}\right\rangle=h(\varepsilon) \delta F_{2}+o(h(\varepsilon)), \\
& J_{\varepsilon}\left(u_{\varepsilon}\right)-J_{0}\left(u_{\varepsilon}\right)=h(\varepsilon) \delta J_{1}+o(h(\varepsilon)), \\
& J_{0}\left(u_{\varepsilon}\right)-J_{0}\left(u_{0}\right)+D J_{0}\left(u_{0}\right)\left(u_{\varepsilon}-u_{0}\right)=h(\varepsilon) \delta J_{2}+o(h(\varepsilon)) .
\end{aligned}
$$

Then we have the asymptotic expansion

$$
\left.J_{\mathcal{\varepsilon}}\left(u_{\varepsilon}\right)-J_{0}\left(u_{0}\right)=f(\varepsilon)\left(\delta F_{1}+\delta F_{2}+\delta J_{1}+\delta J_{2}\right)\right)+o(h(\varepsilon)) .
$$


Proof. In view of equation (4.3), we can write

$$
J_{\mathcal{\varepsilon}}\left(u_{\varepsilon}\right)-J_{0}\left(u_{0}\right)=J_{\mathcal{\varepsilon}}\left(u_{\varepsilon}\right)-J_{0}\left(u_{0}\right)+\left\langle F_{\varepsilon}\left(u_{\varepsilon}\right)-F_{0}\left(u_{0}\right), v_{0}\right\rangle .
$$

Using (4.5)-(4.8), yields

$$
D J_{0}\left(u_{0}\right)\left(u_{\varepsilon}-u_{0}\right)+h(\varepsilon)\left(\delta J_{1}+\delta J_{2}\right)+D F_{0}\left(u_{0}\right)\left(u_{\varepsilon}-u_{0}\right)+h(\varepsilon)\left(\delta F_{1}+\delta F_{2}\right)+o(h(\varepsilon))
$$

From equation (4.4), we obtain the desired result.

Using Proposition 4.1 and following the same lines as in the proof of [2, Thm 3], we can prove the following theorem.

Theorem 4.1. The topological asymptotic expansion of the functional $J_{p}$ is given by

$$
J_{p}\left(u_{\varepsilon}\right)-J_{p}\left(u_{0}\right)=\varepsilon^{2} G\left(x_{0}\right)+o\left(\varepsilon^{2}\right),
$$

where

$$
\begin{aligned}
& G\left(x_{0}\right)=\pi \Lambda \nabla u_{0}\left(x_{0}\right) \cdot \nabla v_{0}\left(x_{0}\right)-c^{c o r e} \pi \delta^{2} u_{0}\left(x_{0}\right) v_{0}\left(x_{0}\right)-f^{c o r e} \pi \delta^{2} v_{0}\left(x_{0}\right), \\
& \Lambda:=\frac{2\left(1-\delta^{2}\right) \sigma^{\text {gaps }}\left(\sigma^{i s o}-\sigma_{\text {gaps }}\right)\left(\sigma^{i s o}+\sigma^{\text {core }}\right)+4 \delta^{2} \sigma^{\text {gaps }} \sigma^{i s o}\left(\sigma^{\text {core }}-\sigma^{\text {gaps }}\right)}{\left(\sigma^{i s o}+\sigma^{\text {core }}\right)\left(\sigma^{\text {iso }}+\sigma^{\text {gaps }}\right)-\delta^{2}\left(\sigma^{i s o}-\sigma^{\text {core }}\right)\left(\sigma^{\text {iso }}-\sigma_{\text {gaps }}\right)}
\end{aligned}
$$

where $u_{0}$ is solution of the problem (1.1) with $\Omega_{k}^{i s o}=\Omega_{k}^{\text {core }}=\varnothing$ and the adjoint state $v_{0}$ is solution of

$$
\begin{gathered}
\int_{\Omega^{e}} \sigma^{e} \nabla v_{0} \cdot \nabla \hat{v} d x+\int_{\Omega^{g a p s}} \sigma^{g a p s} \nabla v_{0} \cdot \nabla \hat{v} d x+\int_{\partial \Omega}\left(\alpha^{\prime}\left(u_{0}\right)\left(u_{0}-u_{a m b}\right)+\alpha\left(u_{0}\right)\right) v_{0} \hat{v} d s \\
+\int_{\Omega} u_{0}\left|u_{0}\right|^{p-2} \hat{v} d x=0, \quad \forall \hat{v} \in H^{1}(\Omega)
\end{gathered}
$$

\section{Algorithm and numerical results}

\subsection{Descent direction}

Definition 5.1. Let $V \in \mathcal{D}^{1}\left(\Omega, \mathbb{R}^{d}\right)$ ( the space of continuous functions with support cmmpact in $\Omega$ ) and denote $T_{t}(x)=x+t V(x)$ the associated transformation. We say that $V$ is a descent direction for a functional $J: \Omega \rightarrow \mathbb{R}$ if there exists $\varepsilon>0$ such that

$$
J\left(T_{t}(\Omega)\right)<J(\Omega) \quad \forall t \in(0, \varepsilon) .
$$

If the Eulerian semiderivative of $J$ at $\Omega$ in direction $V$ exists and if it is a descent direction then by definition

$$
d J(\Omega ; V)<0 .
$$


We use descent descent directions in iterative methods to find a possible local minimizers of the functional $J_{p}$. The strategy is to start with initial shape $\Omega$ and compute descent direction $V$, then we proceed a long this direction as long as the cost functional $J_{p}$ reduces sufficiently using a line search strategy.

In our problem the shapes $\omega_{k}$ are known and invariant under a rotation but their locations are unknown. It is meaningful to use translations to move the shapes $\omega_{k}$.

One may choose a velocity $V$ as a piecewise linear function so that $V$ is a translation on $\Gamma_{k}^{i}, \Gamma_{k}^{e}$ and vanish on $\Omega^{e}$. In order to obtain transformation which is locally a translation one may choose the class of vector fields $V=\left(b_{k}^{1} \zeta, b_{k}^{2} \zeta\right)^{T}:=\left(V_{k}^{1}, V_{k}^{2}\right)^{T}$, where $b_{k}^{1}, b_{k}^{2} \in \mathbb{R}$ and $\zeta$ is a smooth function equal to one in a neighborhood $\omega_{k}^{*}$ of $\omega_{k}$ and equal to zero in $\Omega^{e}$.

The boundary expression of the shape derivative can be written as

$$
d J_{p}(\Omega ; V)=\sum_{k=1}^{N} \int_{\Gamma_{k}^{i}} g_{k} V \cdot n d s+\sum_{k=1}^{N} \int_{\Gamma_{k}^{e}} h_{k} V \cdot n d s
$$

On the boundaries $\Gamma_{k}^{i}, \Gamma_{k}^{e}, V=\left(b_{k}^{1}, b_{k}^{2}\right)^{T}$. Then plugging $V$ with $n=\left(n_{k}^{1}, n_{k}^{2}\right)^{T}$ in the above expression, one obtains

$$
d J(\Omega ; V)=\sum_{k=1}^{N} b_{k}^{1} \int_{\Gamma_{k}^{i}} g_{k} n_{k}^{1} d s+\sum_{k=1}^{N} b_{k}^{1} \int_{\Gamma_{k}^{e}} h_{k} n_{k}^{1} d s+\sum_{k=1}^{N} b_{k}^{2} \int_{\Gamma_{k}^{i}} g_{k} n_{k}^{2} d s+\sum_{k=1}^{N} b_{k}^{2} \int_{\Gamma_{k}^{e}} h_{k} n_{k}^{2} d s
$$

To get a descent direction, one may choose

$$
b_{k}^{1}=-\int_{\Gamma_{k}^{i}} g_{k} n_{k}^{1} d s-\int_{\Gamma_{k}^{e}} h_{k} n_{k}^{1} d s \quad \text { and } \quad b_{k}^{2}=-\int_{\Gamma_{k}^{i}} g_{k} n_{k}^{2} d s-\int_{\Gamma_{k}^{e}} h_{k} n_{k}^{2} d s
$$

For our numerical results, we use the boundary expression of the shape derivative to get a descent directions and gradient algorithm with backtracking line search to solve the optimization problem.

To avoid the overlap of the inclusions during the optimization process, we follow the routine presented in [12]. To prevent the exit to the external boundary, we make a constraint on the distance between the center of the inclusions and the center of the domain $\Omega$. Since we use a gradient-based method, we implement a line search to adjust the time-stepping. The algorithm is stopped when the decrease of the functional becomes insignificant.

\subsection{Algorithms}

The numerical procedure, is described in the following algorithms. 
Table 1: Parameter values of the simulations.

\begin{tabular}{|llll|}
\hline Parameter value & Symbol & Value & Unit \\
\hline Heat conductivity of metallic conductors & $\sigma_{k}^{\text {core }}$ & 401 & $\mathrm{~W} /(\mathrm{m} . \mathrm{K})$ \\
Heat conductivity of single cables insulation & $\sigma_{k}^{\text {iso }}$ & 0.19 & $\mathrm{~W} /(\mathrm{m} . \mathrm{K})$ \\
Heat conductivity of outer insulation & $\sigma^{\text {gap }}$ & $2.61 \mathrm{e}-02$ & $\mathrm{~W} /(\mathrm{m} . \mathrm{K})$ \\
Heat conductivity of air & $\sigma^{e}$ & 0.08 & $\mathrm{~W} /(\mathrm{m} . \mathrm{K})$ \\
Electr. resistivity at reference temperature & $\rho_{0, k}$ & $1.71 \mathrm{e}-08$ & $\Omega . m$ \\
Temperature coefficient of electr. resistivity & $\alpha_{\rho, k}$ & $4.04 \mathrm{e}-03$ & $1 / \mathrm{K}$ \\
Emission coefficient & $\varepsilon_{r}$ & 0.93 & \\
Reference temperature & $u_{r e f}$ & 20 & ${ }^{\circ} \mathrm{C}$ \\
Ambient temperature & $u_{a m b}$ & 32 & ${ }^{\circ} \mathrm{C}$ \\
Number of metallic conductors & $n_{k}$ & 50 & \\
Metallic conductors' diameter & $\delta_{k}$ & 0.2 & $\mathrm{~mm}$ \\
\hline
\end{tabular}

Algorithm 1 (One-shot method).

1. Solve the direct problem and the adjoint problem in the safe domain.

2. Compute the topological gradient $G$.

3. Determine the local minima $\left(x_{0}, y_{0}\right)$ where $G$ is the most negative.

Algorithm 2 (Topology and shape gradient strategy).

1. Initialization : Finding $\left(x_{0}, y_{0}\right)$ using Algorithm 1.

2. Iteration: $\left(x_{k}, y_{k}\right)$ being computed

a. compute the state $u_{k}$ and the adjoint state $v_{k}$, then we can evaluate the descent direction (5.1).

b. update the center by the gradient method with backtracking linesearch.

3. Stop test: If $\left|D J_{p}(\omega ; V)\right|$ is smaller than a prescribed threshold, then stop, else return to step (2) with $k=k+1$.

\subsection{Numerical results}

In the following examples, we consider realistic values of the physical parameters as in [9]. The parameter values of the simulations are presented in Table 1. In our problem, 
Temperature, Steady state solution

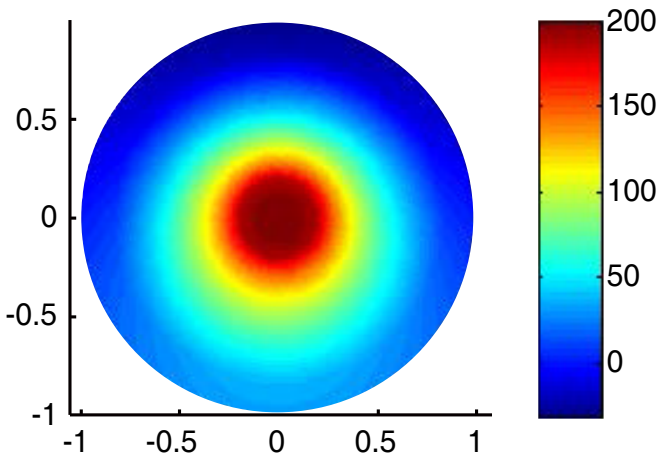

(a)

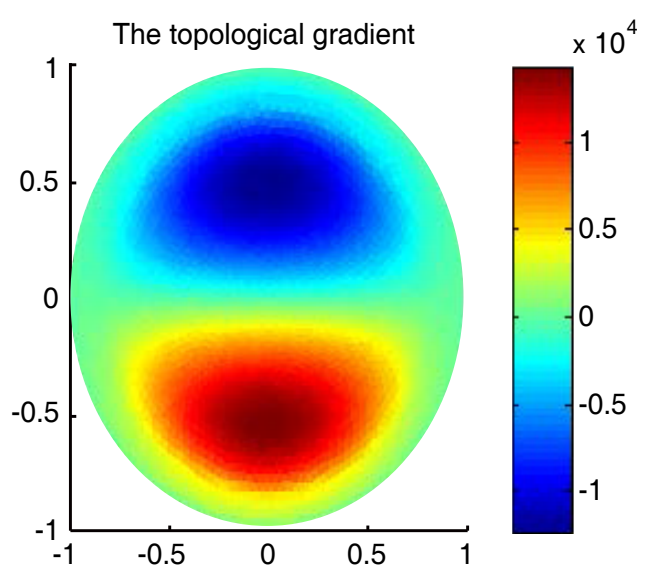

(b)

Figure 2: (a) the initial configuration of temperature (b) the topological derivative of $J_{2}$.

the non linear heat transfer coefficient $\alpha$ is given by the expression (1.5) where $\alpha_{d}$ and $\alpha_{u}$ are explicitly expressed in [12].

\subsection{Example 1}

In this example, we optimize the position of one single cable using Algorithm 2.

The initial coated inclusion is composed of two concentric disks $\Omega^{i s o}$ and $\Omega^{\text {core }}$ with radius $r_{1}=0.2$ and $r_{2}=0.1$ centered at the point $(0.00203,0.49036)$, where the topological gradient is most negative (cf. Figures 3.a)). The optimal position given by the algorithm is $\left(2.90 e^{-05}, 0.49033\right)$ (cf. Figures 3.b)).

\subsection{Example 2}

In this example, we optimize the positions of identical three and four cables respectively using the shape gradient algorithm.

We remark that during the optimization process the inclusions go toward the boundary. Also from figures 4 and 5 we remark that the maximal temperature decrease from $300^{\circ} \mathrm{C}$ to $130^{\circ} \mathrm{C}$ in the case of three cables and from $326^{\circ} \mathrm{C}$ to $122^{\circ} \mathrm{C}$ in the case of four ones.

\subsection{Example 3}

In this example, we optimize the positions of identical four cables using the topological and shape gradient algorithm. The iterative algorithm using the expression (3.21) gives a local minima while the one shot algorithm using the expression (4.10) gives a global minima independent of the choice of the initial positions around the multicable's center. 


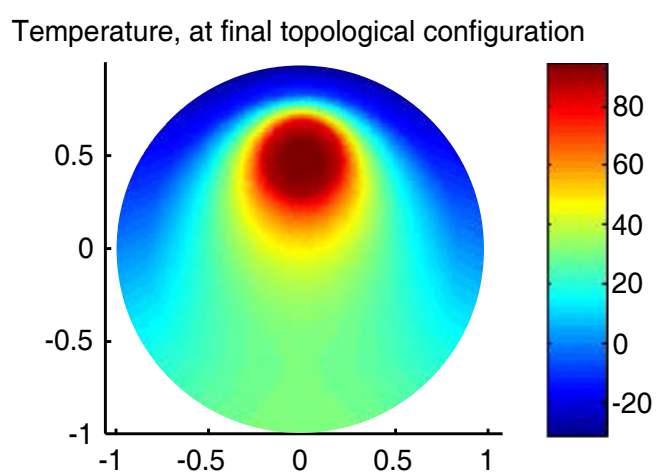

(c)

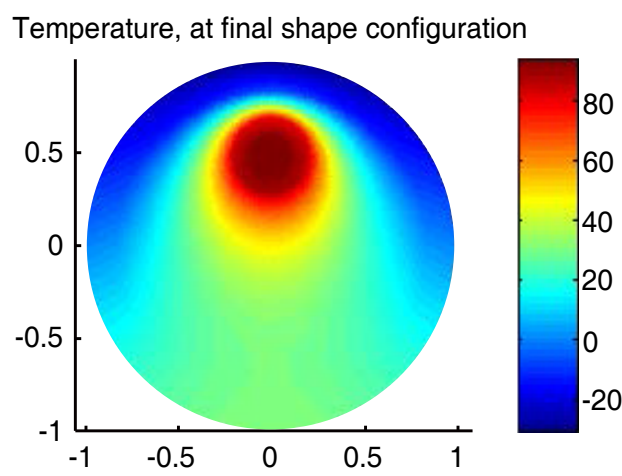

(d)

Figure 3: (c) the final configuration of temperature by the topological derivative and (d) the final configuration of the temperature using Algorithm 1 with $p=2$.

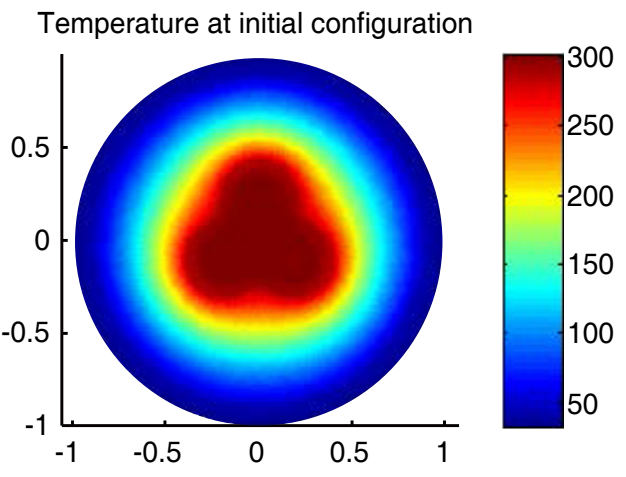

(e)

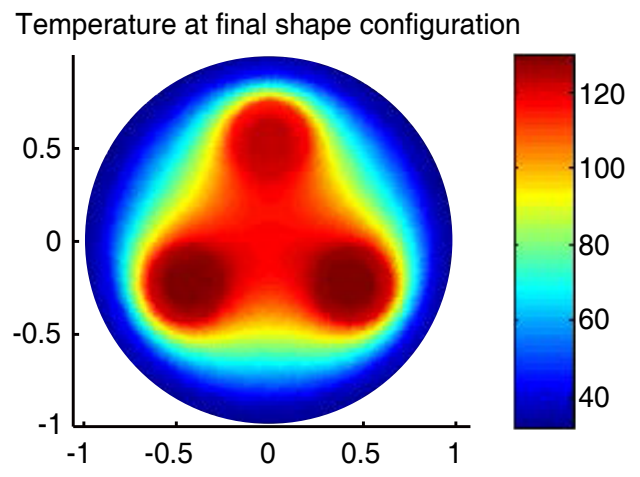

(f)

Figure 4: (e) the temperature distribution of the initial configuration and (f) the temperature distribution for the final configuration of $J_{10}$ for three single cables.

From figure (7) we remark that the use of the iterative method decrease the temperature from $290.62^{\circ} \mathrm{C}$ to $79^{\circ} \mathrm{C}$ and to $71.87^{\circ} \mathrm{C}$ using the one shot method.

Remark 5.1. When $p$ is large enough, the functional $J_{p}$ approximate well the $L^{\infty}$-norm, which is the suitable functional to minimize the maximum temperature. From the numerical point of view, we didn't get a good approximation of the multi-cables when $p$ is large.

For the numerical simulation, we have used two different values of the parameter $p, p=2$ and $p=10$. Although that with this values the functional $J_{p}$ does not approxi- 


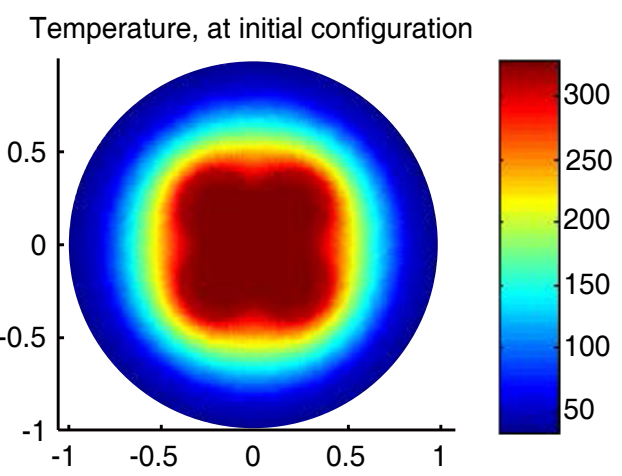

(g)

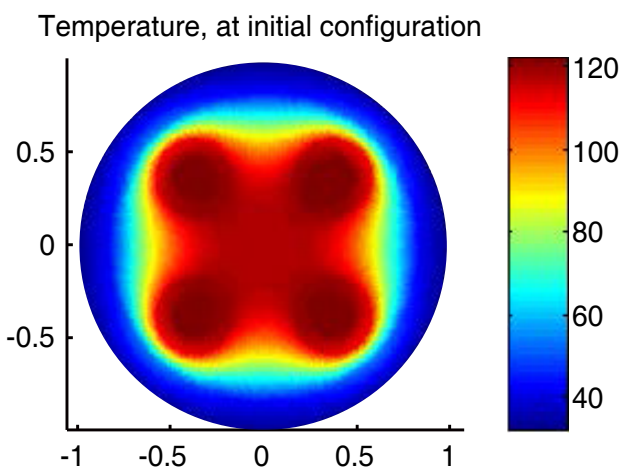

(h)

Figure 5: $(\mathrm{g})$ the temperature distribution of the initial configuration and $(\mathrm{h})$ the temperature distribution for the final configuration of $J_{10}$ for four multicables.

Temperature, Steady state solution

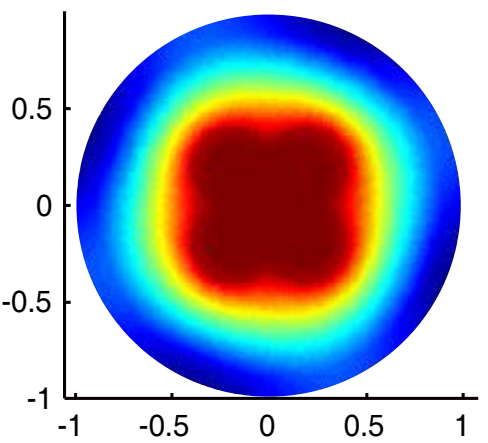

(i)

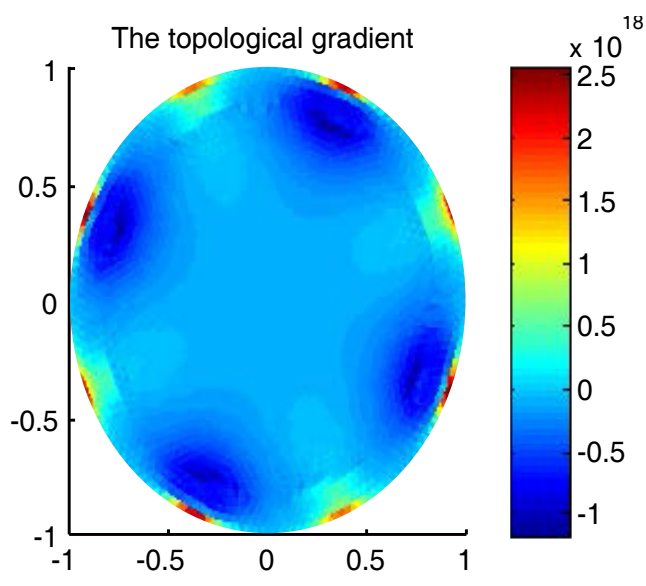

(j)

Figure 6: (i) the temperature distribution of the initial configuration and (j) the topological derivative of $J_{10}$.

mate the $L^{\infty}$-norm very accurately, the maximum temperature is lower than at the initial configuration for the examples presented in this paper.

\section{Appendix}

\subsection{An abstract differentiability result}

In this section, we give an abstract result for differentiating Lagrangian functionals with respect to a parameter. This result is used to prove Theorem 3.2. We first introduce some 


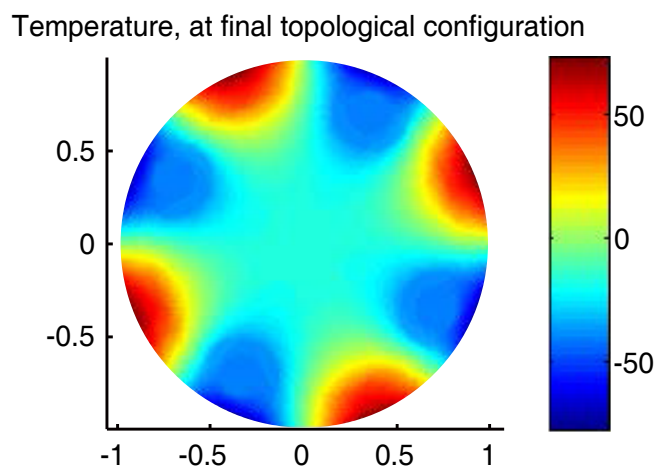

(k)

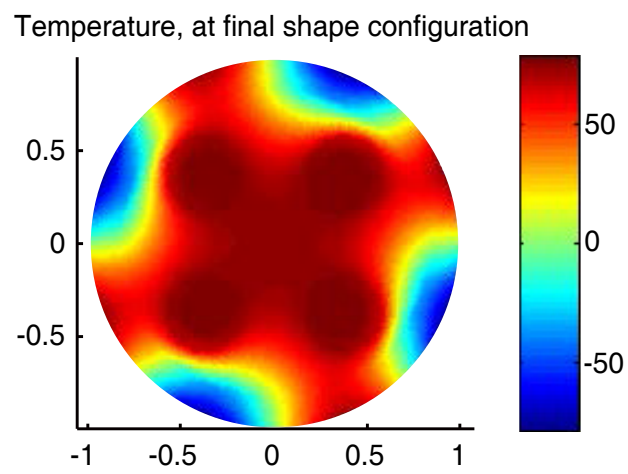

(1)

Figure 7: $(\mathrm{k})$ the temperature distribution at the final configuration given by $(4.10)$ and $(\mathrm{I})$ the temperature distribution at the final configuration given by (3.21)

notations. Consider the functional

$$
G:[0, \varepsilon] \times X \times Y \rightarrow \mathbb{R}
$$

for some $\varepsilon>0$ and the Banach spaces $X$ and $Y$. For each $t \in[0, \varepsilon]$, define

$$
g(t)=\inf _{x \in X} \sup _{y \in Y} G(t, x, y), \quad h(t)=\sup _{y \in Y} \inf G(t, x, y),
$$

and the associated sets

$$
\begin{aligned}
& X(t)=\left\{x^{t} \in X: \sup _{y \in Y} G\left(t, x^{t}, y\right)=g(t)\right\}, \\
& Y(t)=\left\{y^{t} \in Y: \inf _{x \in X} G\left(t, x, y^{t}\right)=h(t)\right\} .
\end{aligned}
$$

Note that inequality $h(t) \leq g(t)$ holds. If $h(t)=g(t)$ the set of saddle points is given by

$$
S(t):=X(t) \times Y(t) .
$$

We state now a simplified version of a result from [6] derived from [5] which gives realistic conditions that allows to differentiate $g(t)$ at $t=0$. The main difficulty is to obtain conditions which allow to exchange the derivative with respect to $t$ and the inf-sup in (6.2).

Theorem 6.1 (Correa and Seeger [5,7]). Let X,Y,G and $\varepsilon$ be given as above. Assume that the following conditions hold: 
(H1) $S(t) \neq \varnothing$ for $0 \leq t \leq \varepsilon$.

(H2) The partial derivative $\partial_{t} G(t, x, y)$ exists for all $(t, x, y) \in[0, \varepsilon] \times X \times Y$.

(H3) For any sequence $\left\{t_{n}\right\}_{n \in \mathbf{N}}$, with $t_{n} \rightarrow 0$, there exist a subsequence $\left\{t_{n_{k}}\right\}_{k \in \mathbf{N}}$ and $x^{0} \in X(0)$, $x_{n_{k}} \in X\left(t_{n_{k}}\right)$ such that for all $y \in Y(0)$,

$$
\lim _{t \searrow 0, k \rightarrow \infty} \partial_{t} G\left(t, x_{n_{k}}, y\right)=\partial_{t} G\left(0, x^{0}, y\right) .
$$

(H4) For any sequence $\left\{t_{n}\right\}_{n \in \mathbf{N}}$, with $t_{n} \rightarrow 0$, there exist a subsequence $\left\{t_{n_{k}}\right\}_{k \in \mathbf{N}}$ and $y^{0} \in Y(0)$, $y_{n_{k}} \in Y\left(t_{n_{k}}\right)$ such that for all $x \in X(0)$,

$$
\lim _{t \searrow 0, k \rightarrow \infty} \partial_{t} G\left(t, x, y_{n_{k}}\right)=\partial_{t} G\left(0, x, y^{0}\right) .
$$

Then there exists $\left(x^{0}, y^{0}\right) \in X(0) \times Y(0)$ such that

$$
\frac{d g}{d t}(0)=\partial_{t} G\left(0, x^{0}, y^{0}\right)
$$

\section{Acknowledgment}

The authors thank the referee for their helpful remarks and comments, which improve the quality of the paper.

\section{References}

[1] S. Amstutz. Sensitivity analysis with respect to a local perturbation of the material property. Asymptotic Anal., 49(1):87-108, 2006.

[2] Z. Belhachmi, A. Ben Abda, B. Meftahi and et al. Topology optimization method with respect to the insertion of small coated inclusion. Asymptotic Anal., 106(2):99-119, 2018.

[3] Z. Belhachmi and H. Meftahi. Shape sensitivity analysis for an interface problem via minimax differentiability. Appl. Math. Comput., 219(12):6828-6842, 2013.

[4] S. Chaabane, M. Masmoudi and H. Meftahi. Topological and shape gradient strategy for solving geometrical inverse problems. J. Math. Anal. Appl., 400(2):724-742, 2013.

[5] R. Correa and A. Seeger. Directional derivative of a minimax function. Nonlinear Anal., 9(1):13-22, 1985.

[6] M. C. Delfour and J.-P. Zolésio. Shape sensitivity analysis via min max differentiability. SIAM J. Control Optim., 26(4):834-862, 1988.

[7] M. C. Delfour and J.-P. Zolésio. Shapes and geometries, volume 22 of Advances in Design and Control. Society for Industrial and Applied Mathematics (SIAM), Philadelphia, PA, second edition, 2011. Metrics, analysis, differential calculus, and optimization.

[8] I. Ekeland and R. Temam. Analyse convexe et problèmes variationnels. Dunod, 1974. Collection Études Mathématiques.

[9] H. Harbrecht and F. Loos. Optimization of current carrying multicables. Comput. Optimization Appl., 63(1):237-271, 2016. 
[10] A. Laurain and K. Sturm. Distributed shape derivative via averaged adjoint method and applications. ESAIM: Math. Model. Numerical Anal., 50(4):1241-1267, 2016.

[11] F. Loos. Joule Heating in Connecting Structures of Automotive Electric Devices-Modelling, Simulation and Optimization. PhD thesis, Universitätsbibliothek der Universität der Bundeswehr München, 2014.

[12] F. Loos, K. Dvorsky and H.-D. Liess. Two approaches for heat transfer simulation of current carrying multicables. Math. Comput. Simulation, 101:13-30, 2014.

[13] M. Masmoudi. The topological asymptotic, in computational methods for control applications, H. Kawarada and J. Periaux, eds, gakuto internat. Ser. Math. Sci. Appli. Gakkotosho, Tokyo, 2002.

[14] H. Meftahi. Stability analysis in the inverse Robin transmission problem. Math. Methods Appl. Sci., 40(7):2505-2521, 2017.

[15] A. A. Novotny, R. A. Feijóo, E. Taroco and et al. Topological sensitivity analysis. Comput. Methods Appl. Mechanics Engin., 192(7):803-829, 2003.

[16] A. Schumacher. Topologieoptimierung von bauteilstrukturen unter verwendung von lochpositionierungkriterien. These de doctorat, Universitat-Gesamthochschule-Siegen, Siegen, 1995.

[17] J. Sokolowski and A. Zochowski. On the topological derivative in shape optimization. SIAM J. Control Optimization, 37(4):1251-1272, 1999.

[18] J. Sokołowski and J.-P. Zolésio. Introduction to shape optimization, volume 16 of Springer Series in Computational Mathematics. Springer-Verlag, Berlin, 1992. Shape sensitivity analysis.

[19] M. S. Vogelius and D. Volkov. Asymptotic formulas for perturbations in the electromagnetic fields due to the presence of inhomogeneities of small diameter. ESAIM: Math. Model. . Numerical Anal., 34(04):723-748, 2000. 